\title{
Fidelity Susceptibility Made Simple: A Unified Quantum Monte Carlo Approach
}

\author{
Lei Wang, ${ }^{1}$ Ye-Hua Liu, ${ }^{1}$ Jakub Imriška, ${ }^{1}$ Ping Nang Ma, ${ }^{2}$ and Matthias Troyer ${ }^{1}$ \\ ${ }^{1}$ Theoretische Physik, ETH Zurich, 8093 Zurich, Switzerland \\ ${ }^{2}$ Yotcopi Technologies, National University of Singapore, 117576, Singapore \\ (Received 10 March 2015; revised manuscript received 8 June 2015; published 15 July 2015)
}

\begin{abstract}
The fidelity susceptibility is a general purpose probe of phase transitions. With its origin in quantum information and in the differential geometry perspective of quantum states, the fidelity susceptibility can indicate the presence of a phase transition without prior knowledge of the local order parameter, as well as reveal the universal properties of a critical point. The wide applicability of the fidelity susceptibility to quantum many-body systems is, however, hindered by the limited computational tools to evaluate it. We present a generic, efficient, and elegant approach to compute the fidelity susceptibility of correlated fermions, bosons, and quantum spin systems in a broad range of quantum Monte Carlo methods. It can be applied to both the ground-state and nonzero-temperature cases. The Monte Carlo estimator has a simple yet universal form, which can be efficiently evaluated in simulations. We demonstrate the power of this approach with applications to the Bose-Hubbard model, the spin- $1 / 2 X X Z$ model, and use it to examine the hypothetical intermediate spin-liquid phase in the Hubbard model on the honeycomb lattice.
\end{abstract}

DOI: 10.1103/PhysRevX.5.031007

\section{INTRODUCTION}

Phase transitions highlight the beauty of universality, despite the great diversity of nature. For example, one finds a unified description for systems ranging from ultracold bosons $[1,2]$ to magnetic insulators [3-5] on the verge of a phase transition. Phase transitions originate from the competition between different tendencies when a macroscopic system tries to organize itself. Thermal fluctuations can drive classical phase transitions at nonzero temperatures, while quantum phase transitions can occur even at zero temperature because of the competition between noncommuting terms in the quantum-mechanical Hamiltonian $[6,7]$. At the phase transition point, physical observables often exhibit singular behavior. In this respect, phase transitions are the most dramatic manifestation of the laws of statistical and quantum mechanics.

Traditional descriptions of phase transitions are based on low-energy effective theories of local order parameters, which have had enormous success in explaining various phase transitions of superfluids, superconductors [8], and quantum magnets $[9,10]$. However, in recent years, exceptions to this Ginzburg-Landau-Wilson paradigm have emerged [11]. In particular, topological phase transitions [12-14] do not have a local order parameter on either side of

Published by the American Physical Society under the terms of the Creative Commons Attribution 3.0 License. Further distribution of this work must maintain attribution to the author(s) and the published article's title, journal citation, and DOI.
Subject Areas: Computational Physics,

Condensed Matter Physics,

Quantum Physics

the phase transition. Therefore, new theoretical tools are needed to search for and characterize these new quantum phases and phase transitions. Many concepts in quantum information science [15], such as quantum fidelity and quantum entanglement, have proven to be useful $[16,17]$. Having a point of view that is totally different from the traditional condensed matter approach, they do not assume the presence of a local order parameter and thus offer new perspectives of the phase transitions and their universalities.

Specifically, we consider the following one-parameter family of Hamiltonians with a driving parameter $\lambda$ :

$$
\hat{H}(\lambda)=\hat{H}_{0}+\lambda \hat{H}_{1} .
$$

As $\lambda$ changes, the system may go through one or several phase transition(s) because of the competition between $\hat{H}_{0}$ and $\hat{H}_{1}$. The quantum fidelity measures the distance on the manifold of $\lambda$, which is defined as the overlap between the ground-state wave functions at two different values of the driving parameter,

$$
F(\lambda, \epsilon)=\left|\left\langle\Psi_{0}(\lambda) \mid \Psi_{0}(\lambda+\epsilon)\right\rangle\right|,
$$

where $\hat{H}(\lambda)\left|\Psi_{n}(\lambda)\right\rangle=E_{n}(\lambda)\left|\Psi_{n}(\lambda)\right\rangle$, and $n=0$ corresponds to the ground state. Unless otherwise stated, we assume the wave functions are normalized and there is no ground-state degeneracy. It is anticipated that the fidelity will exhibit a dip when the two wave functions are qualitatively different, e.g., when they belong to different phases [18]. This wave function overlap is also related to the Anderson orthogonality catastrophe [19] and the Loschmidt echo in quantum dynamics [20]. 
Since in general the quantum fidelity vanishes exponentially with the system size for a many-body system, it is more convenient to study the change of its logarithm with respect to the driving parameter, called the fidelity susceptibility [21]:

$$
\chi_{F}(\lambda)=-\left.\frac{\partial^{2} \ln F}{\partial \epsilon^{2}}\right|_{\epsilon=0} .
$$

The first-order derivative vanishes because $F$ is at its maximum when $\epsilon=0$. In general, the fidelity susceptibility is an extensive quantity away from the critical point, but it exhibits a maximum or even diverges at the critical point, thus indicating a quantum phase transition [22,23]. Similar to conventional thermodynamic quantities, it also follows a scaling law close to the critical point [22-25], which can be used to extract universal information about the phase transition. An important feature of the fidelity susceptibility is that it can reveal a phase transition without prior knowledge of the local order parameter. This makes it suitable for detection of topological phase transitions [26-29] and Berezinsky-Kosterlitz-Thouless-type transitions [30-32] as well as for tackling challenging cases where an in-depth understanding of the underlying physics is still lacking $[33,34]$. Interestingly, the fidelity susceptibility may also be accessible to experiments [35-37].

Despite its appealing features, the difficulty in calculating the fidelity susceptibility has hindered its use in numerical simulations. Many previous studies were thus limited to the cases where the ground-state wave function overlap could be calculated from the analytical solution, exact diagonalization, or density-matrix renormalizationgroup (DMRG) methods [16].

There are several equivalent formulations of the fidelity susceptibility Eq. (3), which reveal different aspects of the quantity. From a computational point of view, they offer direct ways to calculate the fidelity susceptibility without the need to perform numerical derivatives of the fidelity as in Eq. (2).

(a) Expanding $\left|\Psi_{0}(\lambda+\epsilon)\right\rangle$ for small $\epsilon$, one can cast the definition Eq. (3) into an explicit form [22,38]:

$$
\chi_{F}(\lambda)=\frac{\left\langle\partial_{\lambda} \Psi_{0} \mid \partial_{\lambda} \Psi_{0}\right\rangle}{\left\langle\Psi_{0} \mid \Psi_{0}\right\rangle}-\frac{\left\langle\Psi_{0} \mid \partial_{\lambda} \Psi_{0}\right\rangle}{\left\langle\Psi_{0} \mid \Psi_{0}\right\rangle} \frac{\left\langle\partial_{\lambda} \Psi_{0} \mid \Psi_{0}\right\rangle}{\left\langle\Psi_{0} \mid \Psi_{0}\right\rangle} .
$$

The above form does not assume properly normalized wave functions $\left|\Psi_{0}\right\rangle$. Equation (4) reveals the geometric content of the fidelity susceptibility [22,38], since this expression is the real part of the quantum geometric tensor [39].

(b) Alternatively, one can calculate the first-order perturbation for $\left|\Psi_{0}(\lambda+\epsilon)\right\rangle$ and get

$$
\chi_{F}(\lambda)=\sum_{n \neq 0} \frac{\left|\left\langle\Psi_{n}(\lambda)\left|\hat{H}_{1}\right| \Psi_{0}(\lambda)\right\rangle\right|^{2}}{\left[E_{n}(\lambda)-E_{0}(\lambda)\right]^{2}}
$$

Compared to Eq. (4), Eq. (5) does not contain derivatives but involves all eigenstates and the full spectrum. It explicitly shows that $\chi_{F} \geq 0$ and suggests the divergence of $\chi_{F}$ when the energy gap of the system closes.

(c) Reference [21] views Eq. (5) as the zero-frequency component of a spectral representation; thus, a Fourier transform is performed to obtain an alternative expression,

$\chi_{F}(\lambda)=\int_{0}^{\infty} d \tau\left[\left\langle\Psi_{0}\left|\hat{H}_{1}(\tau) \hat{H}_{1}\right| \Psi_{0}\right\rangle-\left\langle\Psi_{0}\left|\hat{H}_{1}\right| \Psi_{0}\right\rangle^{2}\right] \tau$,

where $\hat{H}_{1}(\tau)=e^{\hat{H} \tau} \hat{H}_{1} e^{-\hat{H} \tau}$. Equation (6) has the form of a linear-response formula and is computationally more friendly than Eq. (4) or Eq. (5). References $[24,25]$ generalize it to nonzero temperature by replacing the integration limit with $\beta / 2$ :

$$
\chi_{F}(\lambda)=\int_{0}^{\beta / 2} d \tau\left[\left\langle\hat{H}_{1}(\tau) \hat{H}_{1}\right\rangle-\left\langle\hat{H}_{1}\right\rangle^{2}\right] \tau,
$$

where $\langle\cdots\rangle$ denotes the thermal average at inverse temperature $\beta$. Besides reducing to Eq. (6) as $\beta \rightarrow \infty$, Eq. (7) is nevertheless a well-defined quantity at nonzero temperatures. It bounds the divergence of an alternative "mixed state" fidelity susceptibility $[40,41]$, which is based on the Uhlmann fidelity $[42,43]$, and both quantities follow the same scaling law close to a quantum critical point [24,25]. In general, the evaluation of Eq. (7) is still a formidable computational task, which requires ad hoc implementation depending on the details of the Hamiltonian. For example, the fidelity susceptibility for twodimensional quantum spin systems is calculated in Refs. [24,25] using a quantum Monte Carlo (QMC) method, while for a one-dimensional quantum spin system, Ref. [44] computed it using the transfermatrix DMRG method. The nontrivial implementation of these specific approaches and the overhead in the calculation still limits the wide applicability of the fidelity susceptibility approach to a broad range of quantum many-body systems.

In this paper, we present a simple yet generic approach to compute the fidelity susceptibility in a large variety of modern quantum Monte Carlo methods, including the continuoustime worldline [45-48] and stochastic series expansion (SSE) [49] methods for bosons and quantum spins, and the diagrammatic determinantal methods for quantum impurity [50-53] and fermion lattice models [54-56]. 
In all cases, the Monte Carlo estimator is generic and the implementations are straightforward. As long as the quantum Monte Carlo simulation is feasible (not hindered by the sign problem), the fidelity susceptibility can be easily calculated. Our finding can boost the investigation of quantum phase transitions from a quantum information perspective and becomes especially advantageous for the exploration of exotic phases beyond the Ginzburg-LandauWilson paradigm.

The organization of the paper is as follows. In Sec. II, we present our estimator for the fidelity susceptibility and discuss its implementations in various quantum Monte Carlo methods. Section III presents derivations of the estimator. In Sec. IV, we demonstrate the power of the fidelity susceptibility approach with applications to various models, including correlated bosons, fermions, and quantum spins, using a variety of quantum Monte Carlo methods. Section V discusses the relation between the zerotemperature and nonzero-temperature estimators for the fidelity susceptibility and compares them to the previous approaches $[24,25]$. We conclude with future prospects in Sec. VI.

\section{RESULTS}

We first present our results on the estimator of the fidelity susceptibility in a general setting, then discuss its implementations in various QMC methods, including the continuous-time worldline [45-48] and diagrammatic determinantal approaches [50-56], and the stochastic series expansion method [49]. In all cases, the fidelity susceptibility can be measured with little effort.

\section{A. Universal covariance estimator}

Many modern QMC methods [45-56] share a unified conceptual framework, namely that the partition function is calculated as a perturbative series expansion for the $\lambda \hat{H}_{1}$ term,

$$
Z=\operatorname{Tr}\left(e^{-\beta \hat{H}}\right)=\sum_{k=0}^{\infty} \lambda^{k} \sum_{\mathcal{C}_{k}} w\left(\mathcal{C}_{k}\right)
$$

where the second summation runs over all the Monte Carlo configurations of a given expansion order $k$. The detailed meaning of the configuration depends on the specific QMC algorithm and will be explained in the next subsection. Figure 1(a) depicts a generic configuration, where the $k$ objects residing on the periodic imaginary-time axis represent the vertices $\lambda \hat{H}_{1}$ in the expansion, with a Monte Carlo weight $\lambda^{k} w\left(\mathcal{C}_{k}\right)$ for this configuration. QMC simulations [45-56] sample the summation over $k$ and $\mathcal{C}_{k}$ on an equal footing. Specific algorithms differ by the detailed form of $w\left(\mathcal{C}_{k}\right)$ and by the sampling schemes. Nevertheless, these QMC methods share a unified framework provided by Eq. (8), which is the only requirement for
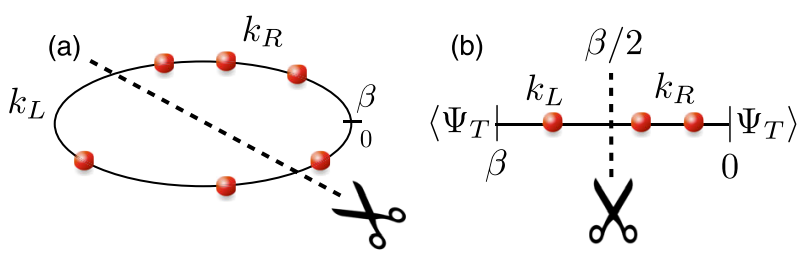

FIG. 1. Measurement of the fidelity susceptibility in the (a) nonzero-temperature formalism and in the (b) ground-state projection scheme. Each red object represents a term in the driving Hamiltonian $\lambda \hat{H}_{1}$, denoted as a vertex. To measure the fidelity susceptibility Eqs. (9) and (11), we divide the imaginarytime axis into two halves and count the number of vertices $k_{L}$ and $k_{R}$, respectively. The nonzero-temperature formalism allows an arbitrary division because of the periodic boundary condition in the imaginary-time axis, while in the ground-state projection scheme the division has to be at $\beta / 2$.

the estimator of the fidelity susceptibility Eq. (7) to possess an appealing universal form in nonzero-temperature QMC simulations:

$$
\chi_{F}^{T \neq 0}=\frac{\left\langle k_{L} k_{R}\right\rangle-\left\langle k_{L}\right\rangle\left\langle k_{R}\right\rangle}{2 \lambda^{2}},
$$

where $k_{L}$ and $k_{R}$ are the number of vertices residing in the range $[\beta / 2, \beta)$ and $[0, \beta / 2)$ of the imaginary-time axis, respectively, shown in Fig. 1(a). The Monte Carlo average of an observable is defined as $\langle O\rangle=\frac{1}{Z} \sum_{k=0}^{\infty} \lambda^{k} \times$ $\sum_{\mathcal{C}_{k}} w\left(\mathcal{C}_{k}\right) O\left(\mathcal{C}_{k}\right)$, where $O\left(\mathcal{C}_{k}\right)$ denotes the value measured for the configuration $\mathcal{C}_{k}$. In practice, because of the periodic boundary condition on the imaginary-time axis, the division of the time axis to halves may be done at an arbitrary location. Moreover, it is even possible to perform multiple measurements on the same configuration by generating several random divisions.

QMC methods [45-56] can also be utilized at zero temperature, where the unnormalized ground-state wave function is obtained from an imaginary-time projection:

$$
\left|\Psi_{0}\right\rangle=\lim _{\beta \rightarrow \infty} e^{-\beta \hat{H} / 2}\left|\Psi_{T}\right\rangle
$$

Here, $\beta$ is a projection parameter and the trial wave funciton $\left|\Psi_{T}\right\rangle$ shall not be orthogonal to the true ground state. A similar framework as Eq. (8) applies, except that one now samples from the overlap $\left\langle\Psi_{T}\left|e^{-\beta \hat{H}}\right| \Psi_{T}\right\rangle$ instead of the partition function. In the projection scheme, the fidelity susceptibility has the estimator

$$
\chi_{F}^{T=0}=\frac{\left\langle k_{L} k_{R}\right\rangle-\left\langle k_{L}\right\rangle\left\langle k_{R}\right\rangle}{\lambda^{2}},
$$

where $k_{L}$ and $k_{R}$ are the number of vertices for the bra and ket states, which reside in the range $[\beta / 2, \beta)$ and $[0, \beta / 2)$ of the imaginary-time axis respectively, shown in Fig. 1(b). 
Since the fidelity susceptibility is non-negative, the covariance formula Eq. (11) reveals positive correlation of $k_{L}$ and $k_{R}$ in a Monte Carlo simulation.

Equations (9) and (11) are the central results of this paper. As is obvious from the discussions in this section, neither the details of the Hamiltonian nor the statistics of the system need to be specified. These estimators are thus general and can be readily implemented in a variety of QMC methods for correlated fermionic, bosonic, or quantum spin systems [45-56].

\section{B. Implementations}

We now discuss implementation of the estimators Eqs. (9) and (11) in various concrete QMC methods. See Sec. II B 1 for discussions about continuous-time worldline [45-48] and diagrammatic determinantal [50-56] approaches and Sec. II B 2 for discussions about the stochastic series expansion approach [49].

\section{Continuous-time worldline and diagrammatic determinantal approaches}

Continuous-time worldline methods [45-48] are widely used to simulate boson and quantum spin systems, while the diagrammatic determinantal approaches are the stateof-the-art methods for solving quantum impurity [50-53] and fermion lattice models [54-56]. A common feature of these methods is to split the Hamiltonian in the form of Eq. (1) and perform a time-dependent expansion in $\lambda \hat{H}_{1}$,

$$
\begin{aligned}
Z= & \sum_{k=0}^{\infty} \lambda^{k} \int_{0}^{\beta} d \tau_{1} \ldots \int_{\tau_{k-1}}^{\beta} d \tau_{k} \\
& \times \operatorname{Tr}\left[(-1)^{k} e^{-\left(\beta-\tau_{k}\right) \hat{H}_{0}} \hat{H}_{1} \ldots \hat{H}_{1} e^{-\tau_{1} \hat{H}_{0}}\right],
\end{aligned}
$$

which obviously fits in the general framework of Eq. (8). In the continuous-time worldline approach [45-48], the $\hat{H}_{1}$ term corresponds to hoppings of bosons or spin flips, depicted as kinks of the worldlines in Fig. 2(a). In continuous-time diagrammatic determinantal approaches
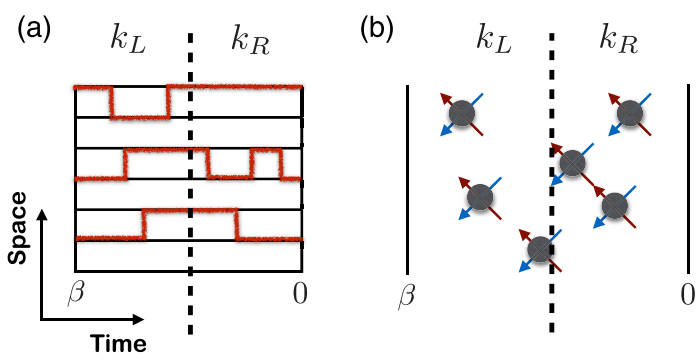

FIG. 2. (a) Measurement of the fidelity susceptibility in a continuous-time worldline QMC simulation of bosons and quantum spins, where one counts the number kinks $k_{L}$ and $k_{R}$ after division of the imaginary-time axis. (b) In the diagrammatic determinantal QMC simulation of correlated fermions, the number of interaction vertices $k_{L}$ and $k_{R}$ are counted.
[50-56], $\lambda \hat{H}_{1}$ contains the fermion interactions, drawn as interaction vertices in Fig. 2(b). Equation (12) has the form of a grand canonical partition function for a classical gas, where $\lambda$ plays the role of fugacity and $k$ is the number of certain classical objects (kinks or vertices) residing on the imaginary-time axis. Typical updates of continuous-time diagrammatic determinantal approaches [50-56] consist of randomly inserting or removing vertices, which are identical to the updates of the grand canonical Monte Carlo method for molecular simulations [57,58]. For bosons and quantum spins there are more effective nonlocal updates, such as the worm and directed loop updates [45-49]. In any case, the Monte Carlo estimators Eqs. (9) and (11) are independent to the detailed sampling procedures. It suffices to count $k_{L}$ and $k_{R}$ of Monte Carlo configurations to calculate the fidelity susceptibility. Examples are presented in Secs. IVA and IV C.

\section{Stochastic series expansion}

SSE is based on a Taylor expansion of the partition function [49],

$$
Z=\sum_{n=0}^{\infty} \frac{(-\beta)^{n}}{n !} \operatorname{Tr}\left[\hat{H}^{n}\right]
$$

which may seem to be different from the framework of Eq. (8). However, as is shown in Ref. [59], one can formally treat the SSE as the time-dependent expansion Eq. (12) with respect to the full Hamiltonian $\hat{H}=\hat{H}_{0}+\lambda \hat{H}_{1}$.

In the implementation of SSE, one truncates the sum to a large number $M$ and pads $M-n$ identity operators in the square bracket of Eq. (13). SSE then samples operators in the fixed-length operator string. To map to a Monte Carlo configuration in the continuous-time formalism, one can assign an imaginary time to each operator, as shown in the bottom of Fig. 3. As long as the mapping keeps the relative order in the original operator string, the Monte Carlo weight remains unchanged [60]. In particular, the configuration is sampled with a weight proportional to $\lambda^{k}$ if there are $k$ of $\lambda \hat{H}_{1}$ operators in the operator string. In this way, although the sampling of SSE is carried out differently from Eq. (12), the general framework of Eq. (8) still applies. The fidelity susceptibility is then measured easily by counting the numbers $k_{L}$ and $k_{R}$ of operators associated with $\lambda \hat{H}_{1}$ in the two halves of the imaginary-time axis after the mapping.

From Fig. 3 it is clear that even though one performs an equal bipartition in the imaginary-time axis, the corresponding location of division is not always in the center of the operator string. In fact, it is easier to directly sample the location of division in the operator string, as shown in the upper part of Fig. 3. A division at the $\ell$ th position $(\ell=0,1, \ldots, M)$ means that there are $\ell$ slots being mapped to one half of the imaginary-time axis and $M-\ell$ slots to 


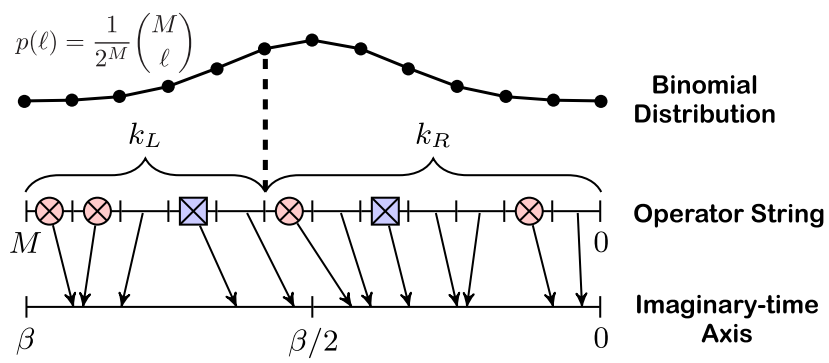

FIG. 3. Division of the operator string in SSE to measure the fidelity susceptibility. The slots represent the fixed-length operator string where empty slots hold identity operators, the red circles (blue squares) correspond to the operators in $\hat{H}_{1}\left(\hat{H}_{0}\right)$. These operators can be mapped to a continuous-time configuration indicated by the arrows. A division can then be made on the imaginary-time axis, for example, at $\beta / 2$. An equivalent approach without explicit mapping to continuous time is to divide the operator string at the location indicated by the vertical dashed line, where the integer $\ell$ is drawn from a binomial distribution. For the estimators Eqs. (9) and (11), one counts the number of red circles (operators in $\lambda \hat{H}_{1}$ ) in both sides for $k_{L}$ and $k_{R}$. In this example, $M=12, n=6, \ell=7$, and $k_{L}=k_{R}=2$.

the other half. Therefore, the division $\ell$ itself follows a binomial distribution $p(\ell)=\frac{1}{2^{M}}\left(\begin{array}{c}M \\ \ell\end{array}\right)$ that can be sampled directly. In this way, the fidelity susceptibility can be efficiently calculated in SSE similar to the continuous-time QMC approaches discussed in Sec. II B 1. As $M \rightarrow \infty$, the binomial distribution approaches a delta function peaked at the center of the operator string. Only in this limit the position in the operator string can be directly interpreted as the imaginary time and a bipartition of the operator string in the center will yield the correct result for the fidelity susceptibility.

\section{DERIVATIONS}

In this section, we derive the estimators for the fidelity susceptibility at nonzero temperature Eq. (9) and for ground-state projector formalism Eq. (11). Readers may skip this section and continue reading with the following sections.

\section{A. Ground state}

Using the diagrammatic expansion for the projection operator, the unnormalized ground-state wave function Eq. (10) has the following form:

$$
\begin{aligned}
\left|\Psi_{0}\right\rangle= & \lim _{\beta \rightarrow \infty} \sum_{k=0}^{\infty} \lambda^{k} \int_{0}^{\beta / 2} d \tau_{1} \ldots \int_{\tau_{k-1}}^{\beta / 2} d \tau_{k} \\
& \times\left[(-1)^{k} e^{-\left(\beta / 2-\tau_{k}\right) \hat{H}_{0}} \hat{H}_{1} \ldots \hat{H}_{1} e^{-\tau_{1} \hat{H}_{0}}\right]\left|\Psi_{T}\right\rangle .
\end{aligned}
$$

Substituting it into Eq. (4) and noticing that the partial derivatives affect only the prefactor $\lambda^{k}$, one obtains Eq. (11). The estimator also holds for the continuous-time auxiliary field expansion methods (CT-AUX [52] and LCTAUX [56]), because one can cast the ground-state wave function to a similar form as Eq. (14), assuming the shift parameter used in these methods [54] to be proportional to $\lambda$.

\section{B. Nonzero temperature}

We present two derivations of the nonzero temperature estimator Eq. (9).

\section{Derivation based on the definition of nonzero-temperature fidelity}

The nonzero-temperature estimator Eq. (9) can be obtained directly from the definition of the nonzerotemperature fidelity [61]:

$$
F=\sqrt{\frac{\operatorname{Tr}\left(e^{-\beta \hat{H}(\lambda) / 2} e^{-\beta \hat{H}(\lambda+\epsilon) / 2}\right)}{\left[\operatorname{Tr}\left(e^{-\beta \hat{H}(\lambda)}\right) \operatorname{Tr}\left(e^{-\beta \hat{H}(\lambda+\epsilon)}\right)\right]^{1 / 2}}}
$$

This is a nonzero-temperature generalization of Eq. (2) and leads to Eq. (7) by using the definition of the fidelity susceptibility Eq. (3) [44]. We expand the traces of the density matrices around the partition function $Z=\operatorname{Tr}\left(e^{-\beta \hat{H}(\lambda)}\right)$ to $O\left(\epsilon^{2}\right)$,

$$
\begin{array}{r}
\operatorname{Tr}\left(e^{-\beta H(\lambda+\epsilon)}\right)=Z+\epsilon \partial_{\lambda} Z+\frac{\epsilon^{2}}{2} \partial_{\lambda}^{2} Z, \\
\operatorname{Tr}\left(e^{-\beta \hat{H}(\lambda) / 2} e^{-\beta H(\lambda+\epsilon) / 2}\right)=Z+\epsilon \vec{\partial}_{\lambda} Z+\frac{\epsilon^{2}}{2} \vec{\partial}_{\lambda}^{2} Z,
\end{array}
$$

where the notation $\vec{\partial}_{\lambda}$ indicates that the partial derivative acts only on operators in the imaginary-time interval $0 \leq \tau<\beta / 2$. Substituting the above two expansions into Eq. (15) and keeping terms up to $O\left(\epsilon^{2}\right)$, one obtains

$$
\begin{aligned}
\chi_{F}^{T \neq 0} & =\frac{\left(\vec{\partial}_{\lambda} Z\right)^{2}}{2 Z^{2}}-\frac{\vec{\partial}_{\lambda}^{2} Z}{2 Z}+\frac{\partial_{\lambda}^{2} Z}{4 Z}-\frac{\left(\partial_{\lambda} Z\right)^{2}}{4 Z^{2}} \\
& =\frac{\left\langle k_{R}\right\rangle^{2}}{2 \lambda^{2}}-\frac{\left\langle k_{R}\left(k_{R}-1\right)\right\rangle}{2 \lambda^{2}}+\frac{\langle k(k-1)\rangle}{4 \lambda^{2}}-\frac{\langle k\rangle^{2}}{4 \lambda^{2}} \\
& =\frac{\left\langle k_{L} k_{R}\right\rangle-\left\langle k_{L}\right\rangle\left\langle k_{R}\right\rangle}{2 \lambda^{2}} .
\end{aligned}
$$

To obtain the second line, we use the partition function expansion Eq. (8) and the fact that the partial derivatives act only on the prefactor $\lambda^{k}=\lambda^{k_{L}+k_{R}}$. For the third line, we use $k=k_{L}+k_{R}$ and $\left\langle k_{L}\right\rangle=\left\langle k_{R}\right\rangle=\langle k\rangle / 2$. This derivation is abstract and is independent of the details of a QMC scheme. Carrying out a similar procedure starting from Eq. (2), one can also prove the ground-state estimator Eq. (11). 


\section{Derivation based on the imaginary-time correlator Eq. (7)}

This derivation starts from the definition of fidelity susceptibility based on the imaginary-time correlator Eq. (7). We utilize its connection to the Monte Carlo weight that appears in Eq. (12) to derive the nonzerotemperature estimator Eq. (9). First of all, the second term in the square bracket of Eq. (7) can be measured directly from the average expansion order $[49,50,53,54]$ :

$$
\left\langle\hat{H}_{1}\right\rangle=-\frac{\langle k\rangle}{\beta \lambda} .
$$

Integrating over the imaginary-time and using $\left\langle k_{L}\right\rangle=$ $\left\langle k_{R}\right\rangle=\langle k\rangle / 2$, one has

$$
\int_{0}^{\beta / 2} d \tau\left[-\left\langle\hat{H}_{1}\right\rangle^{2}\right] \tau=-\frac{\langle k\rangle^{2}}{8 \lambda^{2}}=-\frac{\left\langle k_{L}\right\rangle\left\langle k_{R}\right\rangle}{2 \lambda^{2}} .
$$

We then consider the QMC estimator for the first term of Eq. (7),

$$
\begin{aligned}
G\left(\tau_{1}-\tau_{2}\right) & \triangleq\left\langle\mathcal{T}\left[\hat{H}_{1}\left(\tau_{1}\right) \hat{H}_{1}\left(\tau_{2}\right)\right]\right\rangle \\
& =\frac{1}{\lambda^{2}}\left\langle\sum_{i \neq j} \delta\left(\tau_{i}-\tau_{1}\right) \delta\left(\tau_{j}-\tau_{2}\right)\right\rangle,
\end{aligned}
$$

where $\mathcal{T}$ is the time-ordering operator and $\tau_{i}$ and $\tau_{j}$ are the imaginary times of two vertices in the Monte Carlo configuration. Integrating both sides of Eq. (19) and using the fact that $G\left(\tau_{1}-\tau_{2}\right)$ depends only on $\left|\tau_{1}-\tau_{2}\right|$, one has

$$
\begin{aligned}
\int_{0}^{\Lambda} d \tau_{1} \int_{0}^{\Lambda} d \tau_{2} G\left(\tau_{1}-\tau_{2}\right) & =2 \int_{0}^{\Lambda} d \tau G(\tau)(\Lambda-\tau) \\
& =\frac{1}{\lambda^{2}}\langle k(\Lambda)[k(\Lambda)-1]\rangle,
\end{aligned}
$$

where $k(\Lambda)$ is the number of vertices in the range of $0 \leq \tau<\Lambda$. For example, $k(\beta)=k$ and $k(\beta / 2)=k_{R}$. When choosing $\Lambda=\beta$ and using $G(\tau)=G(\beta-\tau)$, Eq. (20) becomes

$$
\beta \int_{0}^{\beta} d \tau G(\tau)=\frac{1}{\lambda^{2}}\langle k(k-1)\rangle
$$

When setting $\Lambda=\beta / 2$, Eq. (20) reads

$$
2 \int_{0}^{\beta / 2} d \tau G(\tau)\left(\frac{\beta}{2}-\tau\right)=\frac{1}{\lambda^{2}}\left\langle k_{R}\left(k_{R}-1\right)\right\rangle
$$

Together with Eq. (21), it leads to

$$
\begin{aligned}
\int_{0}^{\beta / 2} d \tau G(\tau) \tau & =\frac{\langle k(k-1)\rangle}{4 \lambda^{2}}-\frac{\left\langle k_{R}\left(k_{R}-1\right)\right\rangle}{2 \lambda^{2}} \\
& =\frac{\left\langle k_{L} k_{R}\right\rangle}{2 \lambda^{2}} .
\end{aligned}
$$

In combination with Eqs. (7) and (18), we obtain Eq. (9).

\section{APPLICATIONS}

We first demonstrate the power of the new approach by identifying quantum and thermal phase transitions in the Bose-Hubbard model and in the spin-1/2 XXZ model. Then, we use the fidelity susceptibility to address the presence of the intermediate quantum spin-liquid state in the Hubbard model on the honeycomb lattice. In all cases, this requires only minimal modifications to existing codes. We purposely chose a variety of QMC methods in the following to demonstrate the wide applicability of the covariance estimators. Because of the flexibility of the nonzero-temperature estimator, we use Eq. (9). In Sec. VA, we compare it to the zero-temperature scheme.

\section{A. Quantum phase transition in the Bose-Hubbard model}

First, we use the fidelity susceptibility to probe the quantum phase transition in the Bose-Hubbard model,

$\hat{H}=\sum_{i}\left(\frac{U}{2} \hat{n}_{i}\left(\hat{n}_{i}-1\right)-\mu \hat{n}_{i}\right)-\lambda \sum_{\langle i, j\rangle}\left(\hat{b}_{i}^{\dagger} \hat{b}_{j}+\hat{b}_{j}^{\dagger} \hat{b}_{i}\right)$,

where $U$ is the on-site interaction and $\mu$ is the chemical potential. The driving parameter $\lambda$ has the physical meaning of a tunneling amplitude. The Bose-Hubbard model has a well-known quantum phase transition between the Mott insulating state and the superfluid state as $\lambda / U$ increases $[1,2]$. In particular, for integer fillings, the system has an emergent Lorentz invariance at the critical point and the dynamical critical exponent is $z=1$ [7].

The fidelity susceptibility has previously been calculated using DMRG methods for the one-dimensional BoseHubbard model [62-64]. We now calculate the fidelity susceptibility on a square lattice with $N=L^{2}$ sites at unit filling by tuning $\mu$. In accordance with the dynamical critical exponent $z=1$, we scale the inverse temperature proportionally to the system length $\beta U=4 L$. The simulation employs the directed worm algorithm $[46,65,66]$. We utilize Eq. (9) to sample the fidelity susceptibility by counting the number of kinks in the worldline configuration, as illustrated in Fig. 2(a). Figure 4 shows that, as the system size increases, the peak in the fidelity susceptibility (as a function of the driving parameter $\lambda$ ) is becoming more pronounced around the previously determined critical point $(\lambda / U)_{c}=0.05974(3)$ [67]. The inset of 


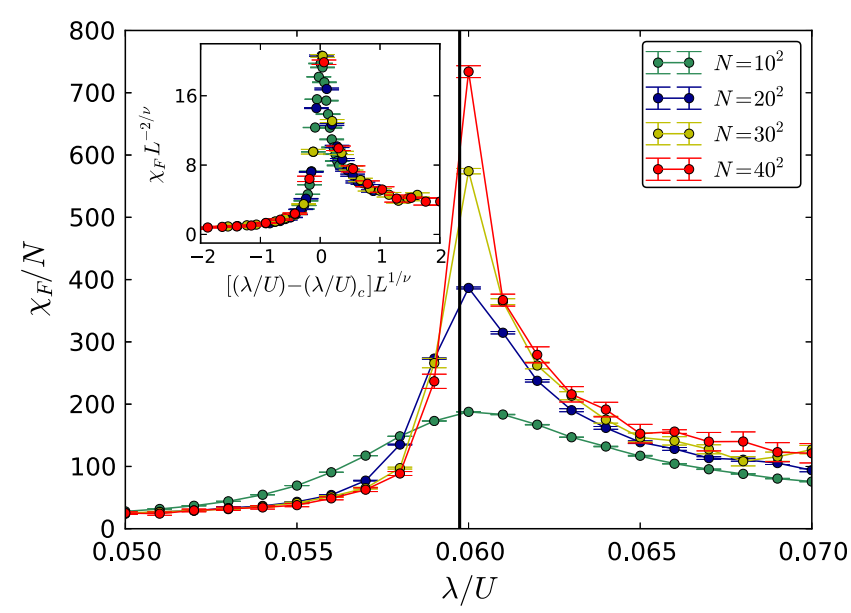

FIG. 4. Fidelity susceptibility per site of a Bose-Hubbard model on a square lattice at unit filling. The vertical line indicates the critical point determined in Ref. [67]. The inset shows the data collapse of the scaled fidelity susceptibility.

Fig. 4 shows the scaled fidelity susceptibility $\chi_{F} L^{-2 / \nu}$ versus $\left[(\lambda / U)-(\lambda / U)_{c}\right] L^{1 / \nu}$ according to the scaling law of Refs. [22,24,25]. The data collapses well under the critical exponent $\nu=0.6715$ of the 3D $X Y$ universality class [67]. The ability to calculate the fidelity susceptibility using the state-of-the-art directed worm algorithm $[46,65,66]$ will greatly advance the study of quantum phase transitions of ultracold bosons. It is worth pointing out that the fidelity susceptibility is related to the quantity (kineticenergy correlator) previously calculated in the study of Higgs mode in a two-dimensional superfluid [68].

\section{B. Thermal phase transition in the $X X Z$ model}

Next, we consider the spin- $1 / 2$ antiferromagnetic $X X Z$ model on a square lattice with $N=L^{2}$ sites,

$$
\hat{H}=J_{z} \sum_{\langle i, j\rangle} \hat{S}_{i}^{z} \hat{S}_{j}^{z}+\lambda \sum_{\langle i, j\rangle}\left(\hat{S}_{i}^{x} \hat{S}_{j}^{x}+\hat{S}_{i}^{y} \hat{S}_{j}^{y}\right)
$$

where the driving parameter $\lambda$ plays the role of the coupling strength in the $X Y$ plane. When $\lambda$ dominates, the Hamiltonian favors Néel order in the $X Y$ plane, while if $J_{z}$ dominates, the system has an antiferromagnetic Ising ground state. The Heisenberg point $\lambda=J_{z}$ is a quantum critical point, which separates the $X Y$ order and the Ising order. This quantum critical point can be easily located from the peak of the fidelity susceptibility (not shown). Our approach makes it possible to obtain the fidelity susceptibility in much larger systems compared to the previous exact diagonalization study [69], and thus can enable a more accurate scaling analysis.

At nonzero temperature, thermal fluctuations will destroy the antiferromagnetic Ising phase at a second-order phase transition. Since one can cross the phase boundary by changing either $\lambda$ or the temperature $T$, we see that the

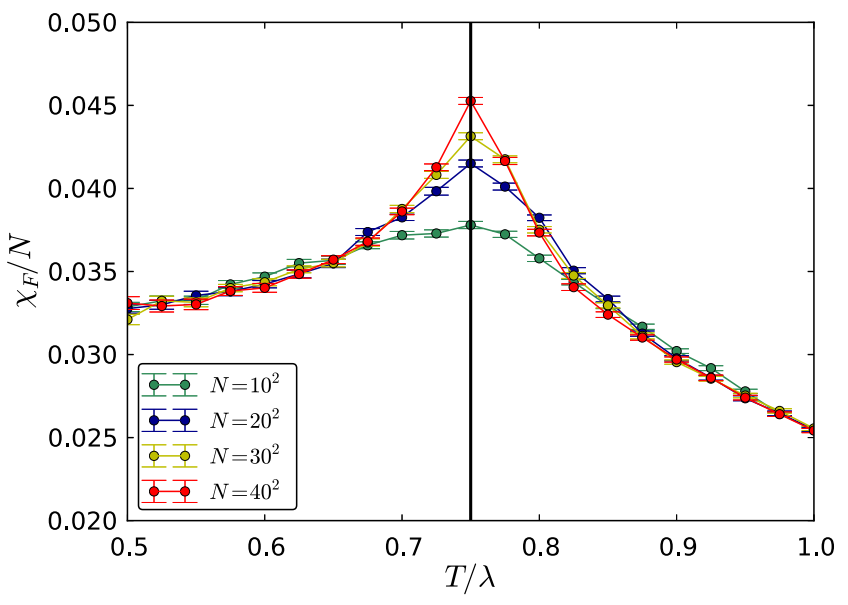

FIG. 5. Fidelity susceptibility per site of a $X X Z$ model on square lattice versus temperature. The vertical line indicates the critical temperature determined in Ref. [70].

fidelity susceptibility can also indicate thermal phase transitions. As a demonstration, we fix $\lambda=1, J_{z}=1.5$ and scan the temperature $T$ to drive a phase transition from the low-temperature antiferromagnetic Ising phase to the high-temperature disordered phase. Figure 5 shows the fidelity susceptibility calculated using Eq. (9) via the SSE method [49,71]. The peak in the fidelity susceptibility correctly singles out the previously determined critical temperature $(T / \lambda)_{c} \approx 0.75[70]$.

\section{Intermediate phase in the Hubbard model on the honeycomb lattice}

Finally, we apply the fidelity susceptibility estimator to a more challenging and controversial example- the Hubbard model on the honeycomb lattice,

$$
\begin{aligned}
\hat{H}= & -t \sum_{\langle i, j\rangle} \sum_{\sigma=\{\uparrow, \downarrow\}}\left(\hat{c}_{i \sigma}^{\dagger} \hat{c}_{j \sigma}+\hat{c}_{j \sigma}^{\dagger} \hat{c}_{i \sigma}\right) \\
& +\lambda \sum_{i}\left(\hat{n}_{i \uparrow}-\frac{1}{2}\right)\left(\hat{n}_{i \downarrow}-\frac{1}{2}\right),
\end{aligned}
$$

where $\lambda$ has the meaning of on-site Hubbard interaction strength. The simulation employs the recently developed efficient continuous-time QMC method for lattice fermions (LCT-INT) [56,72]. We consider lattices with $N=2 L^{2}$ sites, with $L=6,9,12$, and scale the inverse temperature $\beta t=L$.

The ground-state phase diagram of the Hubbard model on the honeycomb lattice [74] has been controversial. It was suggested to possess an intermediate nonmagnetic spin-liquid phase for $\lambda / t \in[3.5,4.3]$ [75]. However, more recent QMC studies on larger systems [76] and with improved observables $[77,78]$ suggest a single continuous phase transition at $\lambda / t \approx 3.8$ belonging to the Gross-Neveu universality class [79]. Other less unbiased methods, such 


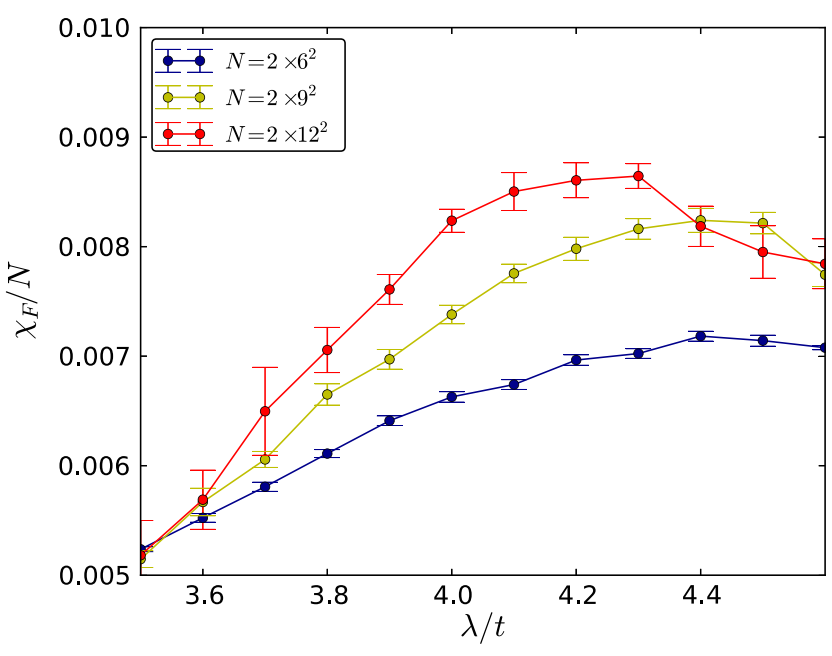

FIG. 6. Fidelity susceptibility per site of the Hubbard model on the honeycomb lattice Eq. (26) with $N=2 L^{2}$ sites.

as quantum cluster approaches, give conflicting results on the presence of the intermediate phase [80-85], depending on implementation details.

The fidelity susceptibility offers a new perspective on the debate about the phase diagram. In the scenario with an intermediate phase, there shall be two features in $\chi_{F}$ when $\lambda / t$ approaches the two phase boundaries. This consideration is independent of the presence of a local-orderparameter description of the possible intermediate phase.

Figure 6 shows the fidelity susceptibility per site for various system sizes obtained using Eq. (9). The calculation of the fidelity susceptibility for fermions is more challenging than that for bosons (Sec. IVA) and quantum spins (Sec. IV B), because of the unsatisfactory local Monte Carlo updates and the cubic (instead of linear) scaling with respect to the system size $N$. Given the limited system sizes, in Fig. 6 we can identify only a single broad peak for small systems (and high temperature). The peak becomes sharper and shifts towards smaller interaction strength as the system size increases; however, the examined system sizes are insufficient for a reliable determination of the phase diagram. Further algorithmic improvements (nonlocal updates and better scaling) on the fermionic QMC methods are needed to access accurate fidelity susceptibility data for larger systems and lower temperatures to better locate phase transitions without knowing the local order parameter. Moreover, by using the histogram reweighting [86,87] or the quantum WangLandau approach [88], it is possible to obtain the fidelity susceptibility in a continuous range of $\lambda$. Such data may be used to precisely determine the critical point and even the critical exponent of the quantum phase transition.

\section{DISCUSSION}

To help the reader gain a better understanding of the estimators Eqs. (9) and (11), we first discuss their relationship then compare them with the previous approach adopted in SSE calculations [24,25]. Finally, we compare the fidelity susceptibility approach with other generic approaches for detecting phase transitions.

\section{A. Relation of the ground-state and nonzero- temperature estimators}

The factor of 2 difference in Eqs. (9) and (11) is due to the different boundary conditions of the imaginary-time axis in the ground-state projection and nonzero-temperature QMC formalisms; see Fig. 1. We use a four-site Hubbard model [Eq. (26)] as an illustrative example. Consider the integrand of Eq. (7), the correlator $G(\tau)=\left\langle\hat{H}_{1}(\tau) \hat{H}_{1}\right\rangle$ is related to the distribution of the vertices on the imaginary-time axis. For a given configuration, the probability of finding two vertices with a time difference $\tau$ is proportional to $\lambda^{2} G(\tau)$. If we equally divide the imaginary-time axis into two halves and impose the additional constraint that the two vertices reside in different halves (denoted as a separable vertex pair), the joint probability changes to $\lambda^{2} G(\tau) \min \{\tau, \beta-\tau\}$. Figure 7(a) shows the histogram of separable vertex pairs accumulated in the imaginary time, which indeed agrees with the exact curve. Summing up the histogram gives the total number of separable vertex pairs, which equals the following integration:

$$
\left\langle k_{L} k_{R}\right\rangle=\lambda^{2} \int_{0}^{\beta / 2} d \tau G(\tau) \tau+\lambda^{2} \int_{\beta / 2}^{\beta} d \tau G(\tau)(\beta-\tau)
$$

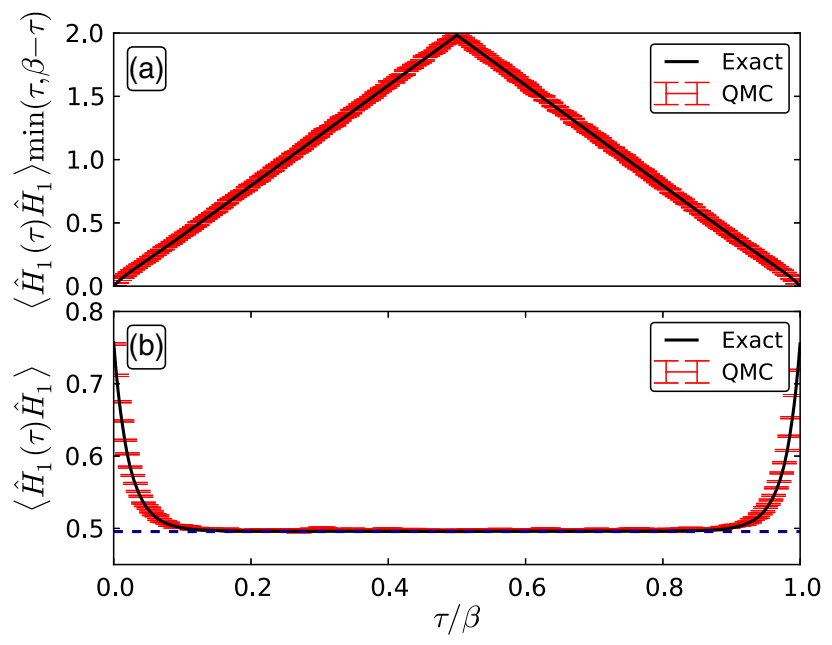

FIG. 7. (a) Histogram obtained by counting separable vertex pairs with distance $\tau$ compared with the exact result of $\left\langle\hat{H}_{1}(\tau) \hat{H}_{1}\right\rangle \max \{\tau, \beta-\tau\}$. (b) Histogram obtained by counting vertices with distance $\tau$ compared with the exact results of $\left\langle\hat{H}_{1}(\tau) \hat{H}_{1}\right\rangle$. For $\tau$ close to $\beta / 2$, the correlator approaches $\left\langle\hat{H}_{1}\right\rangle^{2}$, indicated by the dashed blue line. These simulations are performed for the Hubbard model Eq. (26) on a four-site open chain with $\lambda / t=-2$ and $\beta t=8$. 
Since $G(\tau)$ is symmetric around $\tau=\beta / 2$ in the nonzerotemperature simulation, the two terms of Eq. (27) are equal. Thus, Eq. (27) reduces to Eq. (23). Furthermore, Fig. 7(b) shows the correlator $G(\tau)$ sampled by accumulating the histograms of distances between vertices $[51,53]$ together with the exact results (solid black line). The correlation between vertices decays rapidly with imaginary-time distance and approaches the uncorrelated value $\left\langle\hat{H}_{1}\right\rangle^{2}$ (dashed blue line).

However, in the zero-temperature limit, the correlator $G(\tau)$ decays monotonically with $\tau$ and two vertices will decorrelate for $\tau \geq \beta / 2$, where $\beta \rightarrow \infty$ in the projection scheme. Therefore, the second term of Eq. (27) reduces to $\left(\lambda^{2} \beta^{2}\left\langle\hat{H}_{1}\right\rangle^{2}\right) / 8=\langle k\rangle^{2} / 8$ and cancels half of the second term in the estimator Eq. (11), resolving the apparent difference by the factor of 2 . In practical calculations, it is, however, crucial to adopt the correct formula to obtain consistent results, as illustrated in Fig. 8. The fidelity susceptibility calculated using Eq. (9) in a nonzerotemperature LCT-INT [56] simulation agrees perfectly with exact diagonalization results. The blue square shows the value obtained using Eq. (11) in a projector LCT-INT calculation [89], which correctly reproduces the exact value of the ground-state fidelity susceptibility.

Figure 7(b) also reveals the difficulty of computing the fidelity susceptibility. If the decay of $G(\tau)$ is faster than $1 / \tau$, the integrand of Eq. (7) has vanishing contributions at large $\tau$. However, as the two terms in Eq. (7) are sampled independently in the actual QMC simulations, uncorrelated vertices at large imaginary-time distance will cause noises in the fidelity susceptibility signal. For the applications in Sec. IV, we thus perform the calculations at nonzero temperature as it provides a natural cutoff.

\section{B. Comparison to previous approaches}

The present approach to sample the fidelity susceptibility is more generic and efficient than those developed in Refs. [24,25] specifically for the SSE method. It is

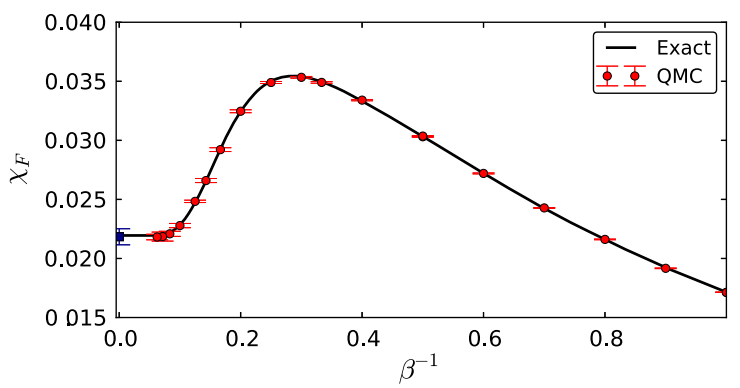

FIG. 8. QMC results for the fidelity susceptibility compared with exact results (solid line). The nonzero-temperature QMC data (red dots) are obtained from Eq. (9), while the ground-state data (blue square at $\beta^{-1}=0$ ) are obtained from Eq. (11) in a projector LCT-INT calculation [89]. The system is the same as in Fig. 7. nevertheless instructive to compare them in detail. The key difference lies in the sampling of the first term of Eq. (7). References [24,25] employ the SSE estimator $[90,91]$

$$
\begin{aligned}
G(\tau)= & \frac{M-1}{\lambda^{2} \beta^{2}} \\
& \times \sum_{n=0}^{M-2}\left(\begin{array}{c}
M-2 \\
n
\end{array}\right)\left(1-\frac{\tau}{\beta}\right)^{M-n-2}\left(\frac{\tau}{\beta}\right)^{n}\langle G(n)\rangle,
\end{aligned}
$$

where $G(n)$ is the number of occurrences of two operators from $\hat{H}_{1}$ that are separated by $n$ positions in the fixedlength operator string ( $n=0$ if they are next to each other). Multiplying both sides with $\max \{\tau, \beta-\tau\}$ and integrating over the imaginary time, one finds

$\lambda^{2} \int_{0}^{\beta} d \tau G(\tau) \max \{\tau, \beta-\tau\}=\sum_{n=0}^{M-2} W(n)\langle G(n)\rangle$

where the weight function $W(n)$ is written in terms of the regularized incomplete beta function $I_{x}(a, b)$ [92],

$$
\begin{aligned}
W(n)= & I_{1 / 2}(n+2, M-n-1) \frac{n+1}{M} \\
& +I_{1 / 2}(M-n, n+1) \frac{M-n-1}{M} .
\end{aligned}
$$

References [24,25] explicitly go through $k(k-1) / 2$ pairs of vertices to accumulate $\langle G(n)\rangle$ and multiply it with the weight function $W(n)$. In Eq. (27), however, the multiplication by the imaginary time $\tau$ is taken into account implicitly by the sampling procedure (which requires separable vertices). Besides being more generic, our approach reduces the computational cost from $\mathcal{O}\left(k^{2}\right)$ to $\mathcal{O}(k)$, which is crucial for the simulation of bosonic and quantum spin systems. In this sense, the specification of our general result Eq. (9) for the SSE method can be regarded as an improved estimator of Eq. (28), which by itself already improves the approach of Refs. [24,25] in several aspects [93]. The improved estimator Eq. (9) not only unifies the SSE approach in a broader context of continuous-time diagrammatic QMC methods, it also gives better statistics with less computational cost compared to Eq. (28).

Figure 9 shows the weight function $W(n)$ for various truncation lengths. As $M$ increases, it approaches two straight lines, and a division in the center of the operator string would yield increasingly accurate results for the fidelity susceptibility, consistent with the discussion in Sec. II B 2 concerning the large $M$ limit.

\section{Relationship to other quantities}

The fidelity susceptibility is related to the second-order derivative of the free energy $A=-(1 / \beta) \ln Z[25,94]$. 


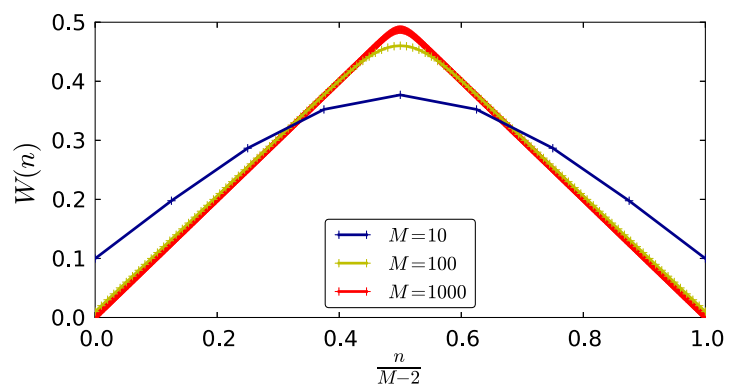

FIG. 9. The weight function according to Eq. (29) for various truncation lengths $M$ in a SSE calculation.

Because of the Hellmann-Feynman theorem [95,96], $\left\langle\hat{H}_{1}\right\rangle$ equals to the first-order derivative of the free energy with respect to $\lambda$. A further derivative following the Kubo formula gives

$$
\begin{aligned}
\frac{\partial^{2} A}{\partial \lambda^{2}}=\frac{\partial\left\langle\hat{H}_{1}\right\rangle}{\partial \lambda} & =-\int_{0}^{\beta} d \tau\left[\left\langle\hat{H}_{1}(\tau) \hat{H}_{1}\right\rangle-\left\langle\hat{H}_{1}\right\rangle^{2}\right] \\
& =\frac{\left\langle k^{2}\right\rangle-\langle k\rangle^{2}-\langle k\rangle}{-\beta \lambda^{2}} .
\end{aligned}
$$

The third equality follows from Eqs. (17) and (21) [97]. The quantity resembles the widely used SSE estimator for the specific heat $[49,98]$, but can be used to probe quantum phase transitions [25]. At zero temperature $\left(\partial\left\langle\hat{H}_{1}\right\rangle\right) / \partial \lambda=-(1 / \lambda)\left(\partial\left\langle\hat{H}_{0}\right\rangle\right) / \partial \lambda$, and the latter quantity is computed using numerical differentiation of the kinetic energy, so as to address the quantum phase transition in the Hubbard model on the honeycomb lattice $[75,85]$. As is pointed out in Refs. [25,94], the fidelity susceptibility has a stronger singularity compared to the second-order derivative of the free energy and is thus a better indicator of quantum phase transitions. In particular, notice that the integrand of Eq. (30) differs from Eq. (7) by a multiplicative factor $\tau$. One can show that in the critical region the singular part of $\chi_{F}$ diverges faster than Eq. (30) by a factor of $L^{z}$, where $z$ is the dynamical critical exponent $[22,25]$. There are concrete examples in a class of topological phase transitions, which do not exhibit singularity in the secondorder derivative of the ground-state energy [99], but can still be detected using the fidelity susceptibility [100].

The covariance that appears in the estimators Eqs. (9) and (11) can also be written as

$$
\left\langle k_{L} k_{R}\right\rangle-\left\langle k_{L}\right\rangle\left\langle k_{R}\right\rangle=\frac{1}{2}\left[\operatorname{Var}(k)-\operatorname{Var}\left(k_{L}\right)-\operatorname{Var}\left(k_{R}\right)\right],
$$

where $\operatorname{Var}(x)=\left\langle x^{2}\right\rangle-\langle x\rangle^{2}$ is the variance of $x$. This expression has an appealing meaning, i.e., the distributions of the vertices residing on the whole and on the halves of the imaginary-time axis have different widths, and the difference in these widths gives the estimate. This form resembles the bipartite fluctuation [101], which was proposed to be a diagnostic tool for phase transitions [102] because of its relation to the entanglement entropy. However, there are important differences. First, the fidelity susceptibility estimator requires a division in the imaginary-time axis for vertices, not in the real space for the physical particles. Second, the total number of vertices is fluctuating in the QMC simulations as opposed to being conserved in the case of bipartition fluctuations. Third, it is easier to locate the critical point using the fidelity susceptibility. As is shown in this paper and in many previous studies [16], the fidelity susceptibility exhibits an increasingly sharp peak at a phase transition as the system size enlarges. On the other hand, to utilize bipartite fluctuations and entanglement entropy for phase transition, one typically needs to resolve the scaling or subleading behavior with the system size, which is often difficult in finite size simulations.

\section{OUTLOOK}

We present a general approach to compute the fidelity susceptibility of correlated fermions, bosons, and quantum spin systems in a broad class of quantum Monte Carlo methods [45-56]. The calculation of the fidelity susceptibility is surprisingly simple yet generic. It provides a general purpose indicator of quantum phase transitions without the need for a prior knowledge of the local order parameter.

Conceptually, our work shows it is rewarding to view the modern QMC methods [45-56] in a unified framework provided by Eq. (8), which deals with the same type of classical statistical problem irrespective of microscopic details of the original quantum system. In the QMC simulations, a quantum phase transition manifests itself as a particle condensation transition driven by changing of the fugacity of the corresponding classical model. This connection suggests generic ways to detect and characterize quantum phase transition through studying classical particle condensations. For example, Eq. (30) actually relates the second-order derivative of free energy of a quantum system to the particle compressibility of a virtual classical system. In this respect, the significance of the covariance estimators Eqs. (9) and (11) is evident because they capture the key critical fluctuation upon a particle condensation transition.

It is straightforward to generalize our Eqs. (9) and (11) to cases with multiple driving parameters, where one needs to count the vertices of different types (as is already done in the SSE calculations in Secs. II B 2 and IV B). It is interesting to find out whether this can lead to a general approach to measure the Berry curvature (the imaginary part of the quantum geometric tensor) in quantum Monte Carlo simulations. Related to these efforts, the nonequilibrium QMC method has been developed in recent years $[103,104]$ to study the nonadiabatic response of quantum systems in imaginary time. In particular, it also 
allows the extraction of the fidelity susceptibility and the Berry curvature $[103,105]$. It would be interesting to compare the nonequilibrium QMC approach [103,104] to the equilibrium one presented in this paper.

Last but not least, the Hamiltonian Eq. (1) has further implications beyond quantum phase transitions. The efficient estimators Eqs. (9) and (11) may also provide useful insights in the simulations of adiabatic quantum computation [106-108] and nonadiabatic quantum dynamics [109,110].

\section{ACKNOWLEDGMENTS}

The authors thank Mauro Iazzi, Sergei Isakov, Lode Pollet, and Hiroshi Shinaoka for helpful discussions. Simulations were performed on the Mönch cluster of Platform for Advanced Scientific Computing (PASC) and on the Brutus cluster at ETH Zurich. We have used ALPS libraries [111] for Monte Carlo simulations and data analysis. The results of the Bose-Hubbard model were obtained using the DWA (directed worm algorithm) application, and the results of the $X X Z$ model were obtained using the DIRLOOP_SSE (stochastic series expansion with directed loop updates) application of the ALPS project. This work was supported by ERC Advanced Grant SIMCOFE and by the Swiss National Science Foundation through the National Center of Competence in Research Quantum Science and Technology QSIT.

[1] M. P. A. Fisher, P. B. Weichman, G. Grinstein, and D. S. Fisher, Boson Localization and the Superfluid-Insulator Transition, Phys. Rev. B 40, 546 (1989).

[2] M. Greiner, O. Mandel, T. Esslinger, T. W. Hänsch, and I. Bloch, Quantum Phase Transition from a Superfluid to a Mott Insulator in a Gas of Ultracold Atoms, Nature (London) 415, 39 (2002).

[3] Ch. Rüegg, B. Normand, M. Matsumoto, A. Furrer, D. F. McMorrow, K. W. Krämer, H. U. Güdel, S. N. Gvasaliya, H. Mutka, and M. Boehm, Quantum Magnets under Pressure: Controlling Elementary Excitations in $\mathrm{TlCuCl}_{3}$, Phys. Rev. Lett. 100, 205701 (2008).

[4] T. Giamarchi, C. Rüegg, and O. Tchernyshyov, BoseEinstein Condensation in Magnetic Insulators, Nat. Phys. 4, 198 (2008).

[5] R. Coldea, D. A. Tennant, E. M. Wheeler, E. Wawrzynska, D. Prabhakaran, M. Telling, K. Habicht, P. Smeibidl, and K. Kiefer, Quantum Criticality in an Ising Chain: Experimental Evidence for Emergent E8 Symmetry, Science 327, 177 (2010).

[6] J. A. Hertz, Quantum Critical Phenomena, Phys. Rev. B 14, 1165 (1976).

[7] S. Sachdev, Quantum Phase Transition (Cambridge University Press, Cambridge, England, 2011).

[8] L. P. Gorkov, Microscopic Derivation of the GinzburgLandau Equations in the Theory of Superconductivity, Sov. Phys. JETP 9, 1364 (1959).
[9] S. Chakravarty, B. I. Halperin, and D. R. Nelson, LowTemperature Behavior of Two-Dimensional Quantum Antiferromagnets, Phys. Rev. Lett. 60, 1057 (1988).

[10] A. V. Chubukov and S. Sachdev, Universal Magnetic Properties of $\mathrm{La}_{2-\delta} \mathrm{Sr}_{\delta} \mathrm{CuO}_{4}$ at Intermediate Temperatures, Phys. Rev. Lett. 71, 169 (1993).

[11] X.-G. Wen, Quantum Field Theory of Many-body Systems: From the Origin of Sound to an Origin of Light and Electrons (Oxford University Press, New York, 2007).

[12] X.-G. Wen and Y.-S. Wu, Transitions between the Quantum Hall States and Insulators Induced by Periodic Potentials, Phys. Rev. Lett. 70, 1501 (1993).

[13] N. Read and D. Green, Paired States of Fermions in Two Dimensions with Breaking of Parity and Time-Reversal Symmetries and the Fractional Quantum Hall Effect, Phys. Rev. B 61, 10267 (2000).

[14] A. Kitaev, Anyons in an Exactly Solved Model and Beyond, Ann. Phys. (Amsterdam) 321, 2 (2006).

[15] M. Nielsen and I. Chuang, Quantum Computation and Quantum Information (Cambridge University Press, Cambridge, England, 2010).

[16] S.-J. Gu, Fidelity Approach to Quantum Phase Transitions, Int. J. Mod. Phys. B 24, 4371 (2010).

[17] J. Eisert, M. Cramer, and M. B. Plenio, Colloquium: Area Laws for the Entanglement Entropy, Rev. Mod. Phys. 82, 277 (2010).

[18] P. Zanardi and N. Paunković, Ground State Overlap and Quantum Phase Transitions, Phys. Rev. E 74, 031123 (2006).

[19] P. Anderson, Infrared Catastrophe in Fermi Gases with Local Scattering Potentials, Phys. Rev. Lett. 18, 1049 (1967).

[20] H. Quan, Z. Song, X. Liu, P. Zanardi, and C. Sun, Decay of Loschmidt Echo Enhanced by Quantum Criticality, Phys. Rev. Lett. 96, 140604 (2006).

[21] W.-L. You, Y.-W. Li, and S.-J. Gu, Fidelity, Dynamic Structure Factor, and Susceptibility in Critical Phenomena, Phys. Rev. E 76, 022101 (2007).

[22] L. C. Venuti and P. Zanardi, Quantum Critical Scaling of the Geometric Tensors, Phys. Rev. Lett. 99, 095701 (2007).

[23] S.-J. Gu and H.-Q. Lin, Scaling Dimension of Fidelity Susceptibility in Quantum Phase Transitions, Europhys. Lett. 87, 10003 (2009).

[24] D. Schwandt, F. Alet, and S. Capponi, Quantum Monte Carlo Simulations of Fidelity at Magnetic Quantum Phase Transitions, Phys. Rev. Lett. 103, 170501 (2009).

[25] A. Fabricio Albuquerque, F. Alet, C. Sire, and S. Capponi, Quantum Critical Scaling of Fidelity Susceptibility, Phys. Rev. B 81, 064418 (2010).

[26] D. Abasto, A. Hamma, and P. Zanardi, Fidelity Analysis of Topological Quantum Phase Transitions, Phys. Rev. A 78, 010301 (2008).

[27] S. Yang, S.-J. Gu, C.-P. Sun, and H.-Q. Lin, Fidelity Susceptibility and Long-Range Correlation in the Kitaev Honeycomb Model, Phys. Rev. A 78, 012304 (2008).

[28] J.-H. Zhao and H.-Q. Zhou, Singularities in Ground-State Fidelity and Quantum Phase Transitions for the Kitaev Model, Phys. Rev. B 80, 014403 (2009). 
[29] S. Garnerone, D. Abasto, S. Haas, and P. Zanardi, Fidelity in Topological Quantum Phases of Matter, Phys. Rev. A 79, 032302 (2009).

[30] M.-F. Yang, Ground-State Fidelity in One-Dimensional Gapless Models, Phys. Rev. B 76, 180403 (2007).

[31] B. Wang, M. Feng, and Z.-Q. Chen, Berezinskii-KosterlitzThouless Transition Uncovered by the Fidelity Susceptibility in the XXZ Model, Phys. Rev. A 81, 064301 (2010).

[32] G. Sun, A. K. Kolezhuk, and T. Vekua, Fidelity at Berezinskii-Kosterlitz-Thouless Quantum Phase Transitions, Phys. Rev. B 91, 014418 (2015).

[33] M. Rigol, B. S. Shastry, and S. Haas, Fidelity and Superconductivity in Two-Dimensional t-j Models, Phys. Rev. B 80, 094529 (2009).

[34] C. J. Jia, B. Moritz, C.-C. Chen, B. S. Shastry, and T. P. Devereaux, Fidelity Study of the Superconducting Phase Diagram in the Two-Dimensional Single-Band Hubbard Model, Phys. Rev. B 84, 125113 (2011).

[35] J. Zhang, X. Peng, N. Rajendran, and D. Suter, Detection of Quantum Critical Points by a Probe Qubit, Phys. Rev. Lett. 100, 100501 (2008).

[36] M. Kolodrubetz, V. Gritsev, and A. Polkovnikov, Classifying and Measuring Geometry of a Quantum Ground State Manifold, Phys. Rev. B 88, 064304 (2013).

[37] S.-J. Gu and W. C. Yu, Spectral Function and Fidelity Susceptibility in Quantum Critical Phenomena, Europhys. Lett. 108, 20002 (2014).

[38] P. Zanardi, P. Giorda, and M. Cozzini, InformationTheoretic Differential Geometry of Quantum Phase Transitions, Phys. Rev. Lett. 99, 100603 (2007).

[39] J. P. Provost and G. Vallee, Riemannian Structure on Manifolds of Quantum States, Commun. Math. Phys. 76, 289 (1980).

[40] P. Zanardi, H. Quan, X. Wang, and C. Sun, Mixed-State Fidelity and Quantum Criticality at Finite Temperature, Phys. Rev. A 75, 032109 (2007).

[41] P. Zanardi, L. C. Venuti, and P. Giorda, Bures Metric over Thermal State Manifolds and Quantum Criticality, Phys. Rev. A 76, 062318 (2007).

[42] A. Uhlmann, The "Transition Probability" in the State Space of $A^{*}$-Algebra, Rep. Math. Phys. 9, 273 (1976).

[43] R. Jozsa, Fidelity for Mixed Quantum States, J. Mod. Opt. 41, 2315 (1994).

[44] J. Sirker, Finite-Temperature Fidelity Susceptibility for One-Dimensional Quantum Systems, Phys. Rev. Lett. 105, 117203 (2010).

[45] B. B. Beard and U.-J. Wiese, Simulations of Discrete Quantum Systems in Continuous Euclidean Time, Phys. Rev. Lett. 77, 5130 (1996).

[46] N. V. Prokof'ev, B. V. Svistunov, and I. S. Tupitsyn, Exact, Complete, and Universal Continuous-Time Worldline Monte Carlo Approach to the Statistics of Discrete Quantum Systems, J. Exp. Theor. Phys. 87, 310 (1998).

[47] H. G. Evertz, The Loop Algorithm, Adv. Phys. 52, 1 (2003).

[48] N. Kawashima and K. Harada, Recent Developments of World-Line Monte Carlo Methods, J. Phys. Soc. Jpn. 73, 1379 (2004).
[49] A. W. Sandvik and J. Kurkijärvi, Quantum Monte Carlo Simulation Method for Spin Systems, Phys. Rev. B 43, 5950 (1991).

[50] A. Rubtsov, V. Savkin, and A. Lichtenstein, ContinuousTime Quantum Monte Carlo Method for Fermions, Phys. Rev. B 72, 035122 (2005).

[51] P. Werner, A. Comanac, L. de' Medici, M. Troyer, and A. Millis, Continuous-Time Solver for Quantum Impurity Models, Phys. Rev. Lett. 97, 076405 (2006).

[52] E. Gull, P. Werner, O. Parcollet, and M. Troyer, Continuous-Time Auxiliary-Field Monte Carlo for Quantum Impurity Models, Europhys. Lett. 82, 57003 (2008).

[53] E. Gull, A. J. Millis, A. I. Lichtenstein, A. N. Rubtsov, M. Troyer, and P. Werner, Continuous-Time Monte Carlo Methods for Quantum Impurity Models, Rev. Mod. Phys. 83, 349 (2011).

[54] S. M. A. Rombouts, K. Heyde, and N. Jachowicz, Quantum Monte Carlo Method for Fermions, Free of Discretization Errors, Phys. Rev. Lett. 82, 4155 (1999).

[55] E. Burovski, N. Prokof'ev, B. Svistunov, and M. Troyer, The Fermi-Hubbard Model at Unitarity, New J. Phys. 8, 153 (2006).

[56] M. Iazzi and M. Troyer, Efficient Continuous-Time Quantum Monte Carlo Algorithm for Fermionic Lattice Models, Phys. Rev. B 91, 241118 (2015).

[57] G. E. Norman and V. S. Filinov, Investigations of Phase Transitions by a Monte-Carlo Method, High Temp. 7, 216 (1969).

[58] D. Frenkel and B. Smit, Understanding Molecular Simulation (Elsevier, New York, 2002).

[59] A. W. Sandvik, R. R. P. Singh, and D. K. Campbell, Quantum Monte Carlo in the Interaction Representation: Application to a Spin-Peierls Model, Phys. Rev. B 56, 14510 (1997).

[60] In practice, one can generate imaginary times randomly in the range of $[0, \beta)$, sort them in ascending order, and assign each one to an operator.

[61] It is different from the Uhlmann fidelity $[42,43]$.

[62] P. Buonsante and A. Vezzani, Ground-State Fidelity and Bipartite Entanglement in the Bose-Hubbard Model, Phys. Rev. Lett. 98, 110601 (2007).

[63] J. Carrasquilla, S. Manmana, and M. Rigol, Scaling of the Gap, Fidelity Susceptibility, and Bloch Oscillations across the Superfluid-to-Mott-Insulator Transition in the One-Dimensional Bose-Hubbard Model, Phys. Rev. A 87, 043606 (2013).

[64] M. Łącki, B. Damski, and J. Zakrzewski, Numerical Studies of Ground-State Fidelity of the Bose-Hubbard Model, Phys. Rev. A 89, 033625 (2014).

[65] L. Pollet, K. Van Houcke, and S. M. A. Rombouts, Engineering Local Optimality in Quantum Monte Carlo Algorithms, J. Comput. Phys. 225, 2249 (2007).

[66] P. N. Ma, Ph.D. thesis, ETH Zurich, 2013.

[67] B. Capogrosso-Sansone, Ş. Söyler, N. Prokof'ev, and B. Svistunov, Monte Carlo Study of the Two-Dimensional Bose-Hubbard Model, Phys. Rev. A 77, 015602 (2008).

[68] L. Pollet and N. Prokof'ev, Higgs Mode in a TwoDimensional Superfluid, Phys. Rev. Lett. 109, 010401 (2012). 
[69] W.-C. Yu, H.-M. Kwok, J. Cao, and S.-J. Gu, Fidelity Susceptibility in the Two-Dimensional Transverse-Field Ising and XXZ Models, Phys. Rev. E 80, 021108 (2009).

[70] G. Schmid, S. Todo, M. Troyer, and A. Dorneich, FiniteTemperature Phase Diagram of Hard-Core Bosons in Two Dimensions, Phys. Rev. Lett. 88, 167208 (2002).

[71] O. F. Syljuåsen and A. W. Sandvik, Quantum Monte Carlo with Directed Loops, Phys. Rev. E 66, 046701 (2002).

[72] In the practical simulation, we use $\lambda<0$. However, because of the particle-hole symmetry, $\chi_{F}$ is symmetric around $\lambda=0$. Additionally, we use $\hat{H}_{1}=\sum_{i}\left[\left(\hat{n}_{i \uparrow}-\frac{1}{2}\right) \times\right.$ $\left.\left(\hat{n}_{i \downarrow}-\frac{1}{2}\right)+\delta^{2}\right][50,73]$, where the constant shift $\delta=0.1$ ensures ergodicity of the Monte Carlo sampling. It has no effect on the results of $\chi_{F}$, but leads to a constant offset in Fig. 7.

[73] F. Assaad and T. Lang, Diagrammatic Determinantal Quantum Monte Carlo Methods: Projective Schemes and Applications to the Hubbard-Holstein Model, Phys. Rev. B 76, 035116 (2007).

[74] S. Sorella and E. Tosatti, Semi-Metal-Insulator Transition of the Hubbard Model in the Honeycomb Lattice, Europhys. Lett. 19, 699 (1992).

[75] Z. Y. Meng, T. C. Lang, S. Wessel, F. F. Assaad, and A. Muramatsu, Quantum Spin Liquid Emerging in Two-Dimensional Correlated Dirac Fermions, Nature (London) 464, 847 (2010).

[76] S. Sorella, Y. Otsuka, and S. Yunoki, Absence of a Spin Liquid Phase in the Hubbard Model on the Honeycomb Lattice, Sci. Rep. 2, 992 (2012).

[77] F. F. Assaad and I. F. Herbut, Pinning the Order: The Nature of Quantum Criticality in the Hubbard Model on Honeycomb Lattice, Phys. Rev. X 3, 031010 (2013).

[78] F. Parisen Toldin, M. Hohenadler, F. F. Assaad, and I. F. Herbut, Fermionic Quantum Criticality in Honeycomb and $\pi$-Flux Hubbard Models: Finite-Size Scaling of Renormalization-Group-Invariant Observables from Quantum Monte Carlo, Phys. Rev. B 91, 165108 (2015).

[79] I. F. Herbut, Interactions and Phase Transitions on Graphene's Honeycomb Lattice, Phys. Rev. Lett. 97, 146401 (2006).

[80] S.-L. Yu, X. C. Xie, and J.-X. Li, Mott Physics and Topological Phase Transition in Correlated Dirac Fermions, Phys. Rev. Lett. 107, 010401 (2011).

[81] W. Wu, S. Rachel, W.-M. Liu, and K. L. Hur, Quantum Spin Hall Insulators with Interactions and Lattice Anisotropy, Phys. Rev. B 85, 205102 (2012).

[82] S. Hassan and D. Sénéchal, Absence of Spin Liquid in Nonfrustrated Correlated Systems, Phys. Rev. Lett. 110, 096402 (2013).

[83] A. Liebsch, Comment on "Absence of Spin Liquid in Nonfrustrated Correlated Systems,” Phys. Rev. Lett. 111, 029701 (2013).

[84] S. R. Hassan and D. Sénéchal, Hassan and Sénéchal Reply, Phys. Rev. Lett. 111, 029702 (2013).

[85] Q. Chen, G. H. Booth, S. Sharma, G. Knizia, and G. K.-L. Chan, Intermediate and Spin-Liquid Phase of the HalfFilled Honeycomb Hubbard Model, Phys. Rev. B 89, 165134 (2014).
[86] A. M. Ferrenberg and R. H. Swendsen, New Monte Carlo Technique for Studying Phase Transitions, Phys. Rev. Lett. 61, 2635 (1988).

[87] A. M. Ferrenberg and R. H. Swendsen, Optimized Monte Carlo Data Analysis, Phys. Rev. Lett. 63, 1195 (1989).

[88] M. Troyer, S. Wessel, and F. Alet, Flat Histogram Methods for Quantum Systems: Algorithms to Overcome Tunneling Problems and Calculate the Free Energy, Phys. Rev. Lett. 90, 120201 (2003).

[89] L. Wang, M. Iazzi, P. Corboz, and M. Troyer, Efficient Continuous-Time Quantum Monte Carlo Method for the Ground State of Correlated Fermions, Phys. Rev. B 91, 235151 (2015).

[90] A. W. Sandvik, A Generalization of Handscomb's Quantum Monte Carlo Scheme-Application to the $1 D$ Hubbard Model, J. Phys. A 25, 3667 (1992).

[91] The original formula was derived for the reduced operator string, i.e., without padding the identity operators. However, the same formula holds as well for the fixed-length operator string.

[92] See, for example, the boost math library http://www.boost .org/doc/libs/1_57_0/libs/math/doc/html/math_toolkit/ sf_beta/ibeta_function.html for definition.

[93] Once the truncation $M$ is fixed in the SSE simulation (after equilibration), one can compute and store the onedimensional array Eq. (29), where $I_{x}(a, b)$ is conveniently calculated using function calls to a numerical library.

[94] S. Chen, L. Wang, Y. Hao, and Y. Wang, Intrinsic Relation between Ground-State Fidelity and the Characterization of a Quantum Phase Transition, Phys. Rev. A 77, 032111 (2008).

[95] H. Hellmann, Einführung in die Quantenchemie (Franz Deuticke, Leipzig, 1937), p. 285.

[96] R. P. Feynman, Forces in Molecules, Phys. Rev. 56, 340 (1939).

[97] The result can also be obtained directly by differentiating Eq. (17) and using Eq. (35) of Ref. [89].

[98] P. Sengupta, A. Sandvik, and R. Singh, Specific Heat of Quasi-Two-Dimensional Antiferromagnetic Heisenberg Models with Varying Interplanar Couplings, Phys. Rev. B 68, 094423 (2003).

[99] Z. Cai, S. Chen, S. Kou, and Y. Wang, Properties of a Class of Topological Phase Transitions, Phys. Rev. B 78, 035123 (2008).

[100] C. N. Varney, K. Sun, M. Rigol, and V. Galitski, Interaction Effects and Quantum Phase Transitions in Topological Insulators, Phys. Rev. B 82, 115125 (2010).

[101] H. F. Song, S. Rachel, C. Flindt, I. Klich, N. Laflorencie, and K. L. Hur, Bipartite Fluctuations as a Probe of ManyBody Entanglement, Phys. Rev. B 85, 035409 (2012).

[102] S. Rachel, N. Laflorencie, H. F. Song, and K. L. Hur, Detecting Quantum Critical Points Using Bipartite Fluctuations, Phys. Rev. Lett. 108, 116401 (2012).

[103] C. De Grandi, A. Polkovnikov, and A. W. Sandvik, Universal Nonequilibrium Quantum Dynamics in Imaginary Time, Phys. Rev. B 84, 224303 (2011).

[104] C.-W. Liu, A. Polkovnikov, and A. W. Sandvik, QuasiAdiabatic Quantum Monte Carlo Algorithm for Quantum Evolution in Imaginary Time, Phys. Rev. B 87, 174302 (2013). 
[105] M. Kolodrubetz, Measuring Berry Curvature with Quantum Monte Carlo, Phys. Rev. B 89, 045107 (2014).

[106] E. Farhi, J. Goldstone, S. Gutmann, J. Lapan, A. Lundgren, and D. Preda, A Quantum Adiabatic Evolution Algorithm Applied to Random Instances of an NP-Complete Problem, Science 292, 472 (2001).

[107] Q. Deng, D. V. Averin, M. H. Amin, and P. Smith, Decoherence Induced Deformation of the Ground State in Adiabatic Quantum Computation, Sci. Rep. 3, 1479 (2013).

[108] S. Boixo, T. F. Rønnow, S. V. Isakov, Z. Wang, D. Wecker, D. A. Lidar, J. M. Martinis, and M. Troyer, Evidence for
Quantum Annealing with More Than One Hundred Qubits, Nat. Phys. 10, 218 (2014).

[109] C. De Grandi, V. Gritsev, and A. Polkovnikov, Quench Dynamics Near a Quantum Critical Point, Phys. Rev. B 81, 012303 (2010).

[110] A. Polkovnikov, K. Sengupta, A. Silva, and M. Vengalattore, Colloquium: Nonequilibrium Dynamics of Closed Interacting Quantum Systems, Rev. Mod. Phys. 83, 863 (2011).

[111] B. Bauer et al., The ALPS Project Release 2.0: Open Source Software for Strongly Correlated Systems, J. Stat. Mech. (2011) P05001. 